\title{
Novel Evolutionary Models and Periodic Charts in p- and q-Individual Chromosomes of Auxiliary Lymph Node and Buccal Cells
}

\author{
P. Mehdipour, ${ }^{1}$ F. Javan, ${ }^{1}$ and M. Atri ${ }^{2,3}$ \\ ${ }^{1}$ Department of Medical Genetics, School of Medicine, Tehran University of Medical Sciences, P.O. Box 14176-13151, Tehran, Iran \\ ${ }^{2}$ Department of Surgery, Day General Hospital, Tehran, Iran \\ ${ }^{3}$ Cancer Institute, Tehran University of Medical Sciences, Tehran, Iran
}

Correspondence should be addressed to P. Mehdipour; mehdipor@tums.ac.ir

Received 9 June 2013; Accepted 14 October 2013

Academic Editor: Dinesh Kumbhare

Copyright (C) 2013 P. Mehdipour et al. This is an open access article distributed under the Creative Commons Attribution License, which permits unrestricted use, distribution, and reproduction in any medium, provided the original work is properly cited.

Signal copy number (SCN) and signal intensity (SI) of subtelomeres (ST) are investigated in auxiliary lymph node (ALN) and buccal (BUC) cells by fluorescence in situ hybridization. The extracted total cell of 38256 and 2309 was, respectively, analyzed from the benign ALN- and BUC-cells of an affected breast cancer patient. The Periodic model was based on ST behavior including normal-, down-, and upregulated clones with diverse SCN. The arm-p/q ratio based signature, as a subtelomeric array, reflects discordance and concordance of ST-behavior within individual chromosomes as a concept of "Individualization of Cells" rather than "Global Insight of Cells". The Periodic charts could be considered as a reliable and refreshable platform through which the cellular evolution could be patterned and characterized. Signature of ST-profile in the BUC and ALN cells and the nature of diverse SCN and SI as quantitative and qualitative value led to modeling the real personalized perspective of cellular evolution. Protein expression of Ki67, Cyclin D1, and Cyclin E was assayed, as a complementary panel. These targets could be applied as the predictive and preventive markers for an early detection at BUC and ALN levels to plan the required managements in the breast cancer patients.

Modeling such signature and mining the subtelomeric signal profiling of individual chromosomes' arms could be translated to a personalized developmental insight in buccal-cells and auxiliary lymph node of breast neoplasm's cells by Novel Periodic Charts.

\section{Introduction}

The reliability of buccal (BUC) cells is due to its sensitivity to different substances used by people who may be affected with cancer or other diseases. In such process, the materials in the BUC are combined with dietary factors, and/or any environmental hazard elements (http://aghealth.nih.gov/ 2002). The mucous-secreting epithelial, known as mucosa, is classified into two types: (1) masticatory (keratinized) and (2) inside layer (nonkeratinized). The masticatory mucosa appears to be thick, with a denser and less vascular connective tissue component. Keratin acts as a shielding domain against different types of stimuli [1]. BUC cells are, morphologically, characterized with their relatively large size, flat shape, nongranular cytoplasm, centerline located nucleus, and large ratio of cytoplasm to nucleus. Such characteristics make BUC a reliable tissue for investigations in cell biology and genetics.

Unmasking the nature of diverse copy number as quantitative value and mode of intensity as qualitative insight could help to track the numerical and structural variations within the personalized perspective at individual buccal- and auxiliary lymph node (ALN) cells.

It was previously published that human germline subterminal DNA is epigenetically hypomethylated, but the mode of methylation in somatic tissues revealed to be de novo $[2,3]$. It was also highlighted that subtelomeric single-copy DNA is diverse for each subtelomere $[4,5]$. Regarding the human genome, the euchromatic regions are proximally encircled by heterochromatin and distally by a telomere consisting of several kilobases of the hexamer repeats [4]. In this paper, 
the terminal repeated region was characterized with $2-15 \mathrm{~kb}$ of (TTAGGG) n repeat sequence, and short repeats (50-250) $\mathrm{n}$ are found to be highly enriched in subtelomeric DNA.

Furthermore, it was published that the human subtelomeric region harbor the mosaic duplicons patchworks [5-7] Such alteration is found to be derived from the terminal chromosome translocations [8]. It was also shown that telomere and subterminal sequences are predisposed to the sister chromatid exchanges [9]. Collectively, subtelomeres are sensitive and predisposed to DNA breakage and repair system; in which an expressive evolution at this genomic territory at neighboring domain of telomeres is a surprising event.

Regarding BUC, there are no available papers on subtelomere region at cellular level. However, telomere length was measured in blood, buccal mucosa, and fibroblasts of patients with inherited bone marrow failure syndromes [10]. BUC sample was also used as a target in oral cancer as well [11]. In these disorders, the median Q-PCR of telomere length (TL) was longer in fibroblast and buccal cells than in blood. They suggested that relative TL is tissue-independent in these syndromes and found significant correlations between blood and fibroblasts, blood and BUC cells, and fibroblasts and BUC cells. These data highlights that short telomeres are not limited to blood or fibroblasts, but are also present in BUC cells. Deletion of $2 \mathrm{q}$ subtelomeric regions was found in children with autism [12].

By considering an association between telomere length with aging and age-related diseases including cancer, the importance of diverse telomere length in Alzheimer's cases was related to the type of sampled tissue [13]. They have observed low TL value in blood cells with further decrease in BUC cells. However, the available paper on subtelomere has rather focused at molecular level and cellular targeting has not been considered.

So far, no systematic evaluating method for tracking the individual subtelomere in human chromosomes is available. Besides, lack of key facts on signal copy number (SCN) and signal intensity (SI) at individual cells and chromosome arms in subtelomere region has delayed the innovation of a progressive avenue towards a personalized and cellular based management. Therefore, it was aimed to investigate the whole set of human chromosomes in the large number of cell population of an affected breast cancer patient by fluorescence in situ hybridization (FISH) analysis. This technique would provide extraordinary and broad information on SCN and SI at ALN level and BUC cells. Such data would comprehensively provide the quantitative and qualitative information about subtelomere in the human somatic domain. Collectively, such required method would promptly pave the way to track the individual subtelomere within the system and developmental cancer cell biology.

Buccal domain could be considered as the sensitive and refreshable biological cells with translational target in which the screening would be accessible as the secondary initial aim after peripheral blood. This domain is capable of being affected by environmental elements as well, powering it as a source in which the predisposing and predicting of neoplastic target including benign and malignancy would be possible. So, it is worthy to identify BUC as an internal control tissue.
The SCN and SI of subtelomere territory are reflective of the functional and developmental machinery within the proliferative cells which have their own characteristics' behavior. Such biological model is created through inheritance and further evolutionary process within the individuals' life. Therefore, the aim of this investigation was to explore the importance of BUC cells as the accessible biological target and mining the nature of subtelomeric SCN and SI markers, creating the novel Periodic charts and modeling the process of evolutionary pathways by focusing on the diverse individual chromosomes' arms, ratio of p/q-, and heterogenic behavior at BUC- and ALN-level in human neoplasm's cells.

\section{Results}

2.1. Descriptive Data. The following basic descriptive data is provided as an evidence to support and highlight the diverse and heterogenic subtelomeric behavior at individual chromosomes' p- and q-arm between BUC and ALN.

According to the logistic regression, ratio of BUC/ALN was highly significant for different factors including week-SI (odds ratio:-6.535, CI 0.000-0.016, $P<0.001$ ), and medium SI (odds ratio: 9.135, CI 170.182-505386.699, $P<0.001$ ). A significant positive correlation was found between one SCN with presence of two SCN $(P=0.043)$; week $(P<0.001)$ and strong $(P<0.001) \mathrm{SI}$; and also between two SCN with week $(P<0.001)$ and medium $(P<0.001)$ SI in buccal cells. Same manner of correlation was also achieved either between week and medium SI $(P<.001)$, or between strong and medium SI.

Significant negative correlation could be also traced between lack of any SCN with presence of one $(P=0.06)$ two SCN $(P<0.001)$ and week $(P<0.001)$ and medium $(P<0.001)$ SI.

Regarding the $\mathrm{p}$-arm, lack of SCN revealed to have a higher significant mean in ALN than in BUC for all chromosomes except number $1,2,12,18$, and $19(P<0.01)$. By one and two SCN, BUC cells had significant higher mean than in ALN in all chromosomes, except for chromosomes 17, 19, and 20 in one SCN, and chromosome 3 in two SCN category, respectively $(P<0.01)$. By three SCN, few chromosomes had higher significant mean in ALN than in BUC $(P<0.01)$. Moreover, week SI revealed to have a higher significant mean in BUC than in ALN for all chromosomes except number $2(P<0.01)$. Considering strong SI, all chromosomes showed higher significant mean in BUC than in ALN, except chromosomes 1 and $17(P<0.01)$. By focusing on the qarm, all chromosomes reflected higher mean of SCN in ALNcells than in BUC-cells, except for chromosome $2(P<0.01)$. All chromosomes revealed significant higher mean of SCN by different categories $(P<0.01)$. All categories of SI in $\mathrm{q}$-arm revealed a higher significant mean in BUC than in ALN for all chromosomes $(P<0.01)$. Focusing on the global chromosomal $\mathrm{p}$ - and q-arms, there was a significant mean subtelomeric SCN and SI between ALN and BUC cells $(P<$ $0.01)$. Scoring of SI revealed a significant higher mean in BUC- than in ALN-cells for all chromosomes except number 17 for strong SI and chromosome X for week SI $(P<0.05 \&$ $P<0.001)$. By referring to the mean difference of $\mathrm{p} / \mathrm{q}$ ratio for 
signal intensity, the week, medium, and strong SI revealed to be significantly higher in ALN than in buccal cells except for chromosomes 18, X; and 4, 5, 7, 18, and 19; and 9, 10, and 18, respectively. In addition, protein expressions of Ki67, cyclin $\mathrm{D} 1$, and cyclin E, as the complementary functional assays, are included to present degree of proliferation and the behavior of key targets within the cell cycle.

2.2. Evolutionary Modeling Insight. The Periodic charts, as an informative, supportive, and directive guide, could lead to providing the novel evolutionary modeling for SCN and SI in BUC- and ALN-cells (Figures 1-10).

\section{Discussion}

3.1. Descriptive Data. Overall, the specific subtelomeric probe has been widely applied in autistic disorders [12], and only the limited publication of single probes, including chromosomes 5, 9 and 7q in haematological myeloid disorders are available $[14,15]$. The main aim of the present work was to find the direction of subtelomeric developmental course between BUC cells as an accessible biological target and ALN cells as a benign neoplastic territory for further evolutionary destination. Our findings indicate that, irrespective of degree of SI, BUC cells are highly potent to be candidate as a bridging tissue between peripheral blood and neoplastic tissue (ALN). In this case, higher mean for week- and strongSI reflects degree of downregulated and upregulated intensity, as qualitative value, which is vice versa in ALN. Besides, ALN with lower mean of different degree of SI is less predisposed to alteration in all degrees of SI. By considering medium SI, BUC cells revealed to have higher mean for normal value of SI. Considering $\mathrm{p}$ and $\mathrm{q}$ arms as a united territory, the present findings could also candidate BUC cells as a reliable predictive and predisposing cellular target.

The ALN cells are characterized by higher mean of p-arm in BUC cells for lacking SCN, having one or two SCN for majority of chromosomes. Only chromosomes 1, 17, 19, and 20 for one SCN and chromosome 3 for two SCN revealed to be, significantly, involved in a diverse developmental course. Regarding three SCN, the predisposed p-arm of chromosomes includes $4,6,7,9,10,16$, and 17 as a group which is mostly involved in developmental course concerning gain of genetic material by displaying an additional signal copy number within ALN cells, but chromosomes 5, 8, 11, 20, and $\mathrm{X}$ could be considered as a reliable target within BUC cells.

Almost all chromosomes are predisposed to structural alteration of $\mathrm{p}$ chromosomal arm in BUC, reflected by different degree of SI and representative of loss and gain of genetic material. These findings could candidate BUC cells as a platform through which either the subtelomeric SCN or SI with its heterogenic behavior is involved in course of cellular development and the evolutionary process.

By targeting q-arm, almost all chromosomes are predisposed to numerical alteration by considering lack of any SCN at ALN level. In contrast, losing genetic material by having only one SCN could be highlighted in BUC cells which make it a reliable candidate for being involved in developmental course of evolution at this accessible domain. It could be concluded that that q-arm of all chromosomes is involved in BUC cells. Moreover, the BUC cells by having higher mean of normal SCN status seem to be stable regarding two SCN than in ALN, but in contrast, the course of evolution is remarkable at neoplastic level by reflecting lower mean in ALN cells.

By considering the structural alteration in q-arm of all individual chromosomes, the subtelomeric SI was remarkably affected in BUC cells which could define it as a reliable screening target for early detection. These findings demonstrate the importance of detectable structural alterations by assaying SI of subtelomeric territory.

By considering the SI of p-arm calculated from positive SCN cells, developmental process seems to be diverse at ALN and BUC levels. Higher mean of week SI in ALN cells reflect the decrease of subtelomeric material for all chromosomes except number 18. The medium SI showed, in contrast, higher mean in BUC cells for all chromosomes with exception of chromosomes $1,12,16$, and 17 which is indicative of a diverse development comparing to the week SI. Regarding the strong SI, the scenario was similar to medium SI with additional exception for chromosomes 2, 4, and 20. These findings define ALN as a predisposed target in direction of downregulated SI, characterized as week signals, and BUC domain as upregulated target known as strong signals.

Considering the subtelomeric SI, q-arm of individual chromosomes is diversely behaved and is reflective of a remarkable involvement of BUC domain as an initiative target by higher mean of ST in different categories by highlighting downregulated and upregulated mode.

By looking at the global chromosomes, heterogenic mean of subtelomeric SCN and SI was found between ALN and BUC cells for either $\mathrm{p}$ - or q-arm. Such diversity is reflective of capability of ALN cells to be evolved; however, BUC cells could be used in an early screening assay. Even though the whole set of chromosomes is examined, a diverse trend could be observed between $\mathrm{p}$ - and q-arms as well, but without any chromosomal specification.

To underline the important role of individual chromosomes, the $\mathrm{p}$ - and $\mathrm{q}$-arms even as united domains show a diverse subtelomeric behavior between ALN and BUC cells. Such finding is even more highlighted in cells with three SCN in limited and specific chromosomes including group one that is, 3, 7, 22, and group 2 including chromosomes 11, 13, 14 , and $\mathrm{X}$. These two groups are separately dominant in ALN and BUC cells, respectively.

It seems that chromosome 14 in all categories of SI, and 15 in both weak and medium SI are predisposed to have higher mean in ALN than in BUC.

In addition, the $\mathrm{p} / \mathrm{q}$ ratio is an important value to diversify two chromosomal arms as two different territories. Regarding cells with one and three SCN, the majority of chromosomes had significant higher mean in ALN than in BUC cells except numbers 8 and 18 and 11, respectively. In cases of cells lacking signal or of having two signals, almost $2 / 3$ rd of chromosomes had higher mean in ALN than in BUC cells. These findings support the diverse subtelomeric SCN in p- and q-arm per individual chromosomes and could be considered as a proof of heterogeneity at ALN and BUC level. However, the diverse manner of mean ratio in individual chromosomes in 
BUC cells could be considered as a personalized predictive value.

Almost all individual chromosomes were revealed to have significant higher mean of $\mathrm{p} / \mathrm{q}$ ratio in ALN-cells than in BUC-cells, except few chromosomes of which chromosome 18 is found to play the most common role. Chromosome $\mathrm{X}$ has remarkable higher mean in BUC cells than in ALN cells in which the $\mathrm{p} / \mathrm{q}$ ratio has been extremely reduced in ALN (Figure 1). This could be considered as an evolutionary process. Collectively, our data on SI is highly heterogenic and approves the diverse behavior of $\mathrm{p}$ - and q-arms.

3.2. Evolutionary Modeling Insight. The subtelomeric signals could be well defined at individual BUC-and ALN-cells which characterize the specific nature of $\mathrm{p}$ - and q-arms per individual chromosomes (Figures 1 and 2). In addition, the Periodic charts facilitate the horizontal and vertical maneuver through cross roads and the course of evolution by which the common and diverse involvement of SCN and SI modes could be achieved for individual chromosomes. Moreover, an interactive harmonic mode could be also observed within the SCN and SI groups.

By defining the value of $\mathrm{p} / \mathrm{q}$ ratio, different categories for SCN and SI in ALN- and BUC-cells could be patterned Figures (4-10). Such signatures as the subtelomeric array illustrate how harmonic specific chromosomes share some characteristics of SCN and/or SI, in other words it reflects discordance and concordance of subtelomeric behavior within individual chromosomes by relying on heterogenic behavior of $\mathrm{p} / \mathrm{q}$ ratio. The present Periodic charts could be considered as a reliable and refreshable platform through which the cellular behavior could be designed and characterized within individual chromosomes.

As a configurative model and developmental scheme of subtelomeric $\mathrm{p} / \mathrm{q}$ ratio per individual chromosomes, a tabular system could be designed. Such Periodic chart is organized by providing two triple rows, containing information on $\mathrm{SCN}$ as a quantitative value and SI as qualitative value, respectively. The upper and lower rows are reflective of periodic version and capable of reassembly or recreation according to more complementary data. Different boxes are informative and reflect the cooperation between different categories of SCN and SI, and also the common interaction between specific chromosomes. As a matter of fact, such Periodic charts could be used as a construction for laddering the status of any cellular target which may react with other molecular and/or cellular properties, or as a sole; this would lead to the vertical and/or horizontal array analyses and translates actions and interactions of different involved elements in cancer cell and molecular biology (Figures 6 and 7). Diverse profiling of p/q ratio in ALN- and BUC-cells could be novelized through these charts. Moreover, the common subtelomeric categories could traced through the vertical and horizontal maneuvering by these charts. Sharing the common manner is either between individual chromosomes or different categories of SCN and SI. These Periodic charts could be, systematically, indicate different groups either for SCN and SI as a sole, or as accompanied machinery.
Chromosome 3 is involved in all categories including normal two SCN, losing either one or both SCN, and also gaining an additional SCN which highlights it as a most predisposed chromosome to be diverse at ALN level. Chromosome 10 by involving in normal clone and losing both SCN translates an extreme aberrant condition at quantitative level or behaving as normal. By considering the elevated mean ratio in BUC, chromosome 11 is not involved in any SCN category which seems to act as a restricted chromosome by comparing to the increased ratio in ALN than in BUC (Figure 8). In this chart, by vertical and horizontal evaluation of the SCN, increasing and decreasing p/q values in ALN and BUC could be compared.

When the p/q ratio is reduced in ALN than in BUC, the SCN loss, either for one or for both SCN, is remarkably severe than when this ratio is increased in ALN (Figure 9). However, the SCN gain is limited and only chromosomes 3 , 5,7 , and 8 seem to have an additional SCN. In contrast, when ALN has higher p/q ratio, no single chromosome had a SCN gain. It seems that, irrespective of the manner of evolution by comparing BUC cells as the index target towards ALN, SCN gain is not an essential event, but chromosomes 3, 5, 7, and 8 provide a complementary panel in which both strong and weak SI are able to alter regulation of genetic machinery within the subtelomeric territory.

As Figure 9 shows, there is no sign of SCN gain for chromosomes 9-12, 16-20 and X within the evolutionary course in ALN by mean of decreasing p/q ratio, but in the downregulated categry (Figure 10), the limited chromosomes were revealed to have strong SI (chromosome 9 and 10) and both weak and strong-SI for chromosome 18. Collectively, chromosomes 9,10 , and 18 seem to be predisposed for being involved in ALN- and BUC-cells. This finding would highlight the importance of qualitative factors which could be related to the nature of molecular alteration of the involved gene(s) in subtelomere territory, which may be named as a "target tracing" for molecular analysis. Collectively, evolutionary model of subtelomeric SCN through buccal- and auxiliary lymph node-cells is achieved. Moreover, by considering the BUC as a reliable and index biologic target, increased and decreased SCN in ALN cells are both considered as an evolutionary process.

The chromosomes by having two normal SCN are the initial departure line and could be considered as a baseline, and further alterations including lacking $\mathrm{SCN}$, losing one signal, and gaining one signal are included in the course of evolution. By focusing on the $\mathrm{p} / \mathrm{q}$ ratio and excluding groups D and $\mathrm{G}$ (due to lack of p-arm), chromosomes 120 and $\mathrm{X}$ all by having 2 SCN participated as normal clone in this symphony. Furthermore, irrespective of considering the mode of alteration in SCN-p/q ratio, that is, decline and elevation in BUC- to ALN-cells, all chromosomes, by losing one or both SCN, are involved in the next step of evolution as well. But when the $\mathrm{p} / \mathrm{q}$ ratio of SCN is increased in ALN, the mode of evolution reflects more specific alterations. In this regard, chromosomes $1,2,5,16,17$, and 19 are classified as being involved in normal clone; chromosome 1 is also predisposed to lose both normal SCN; interestingly, chromosomes 3, 4, 17, and 20 are not involved in any SCN 


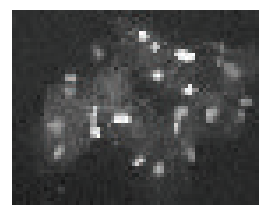

(a)

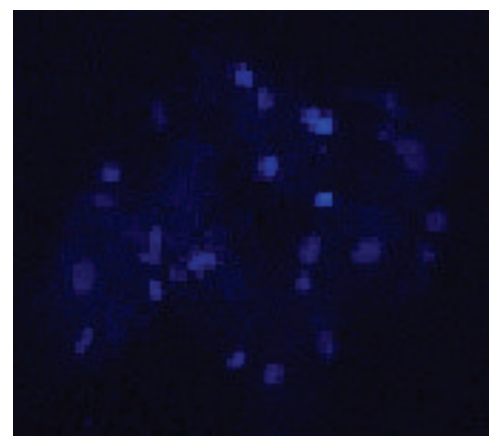

1

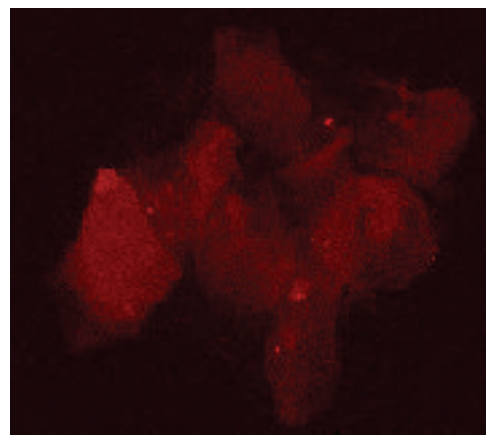

1

(c)

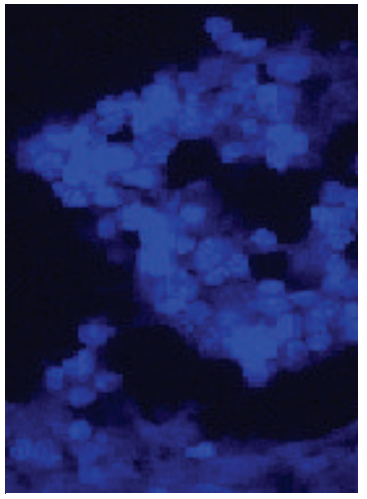

(e)

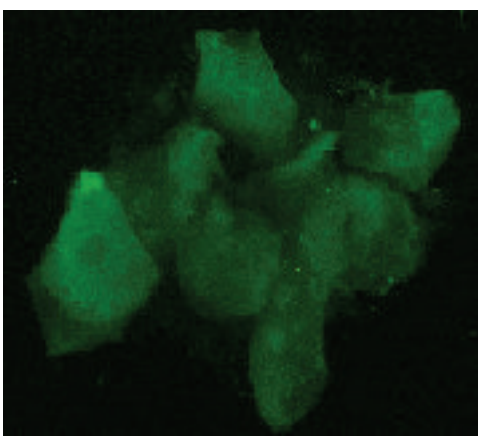

2

(b)

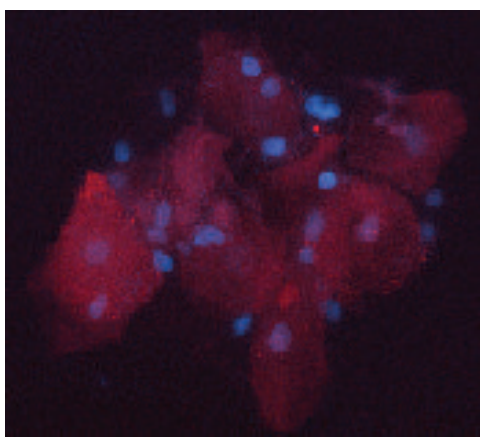

2

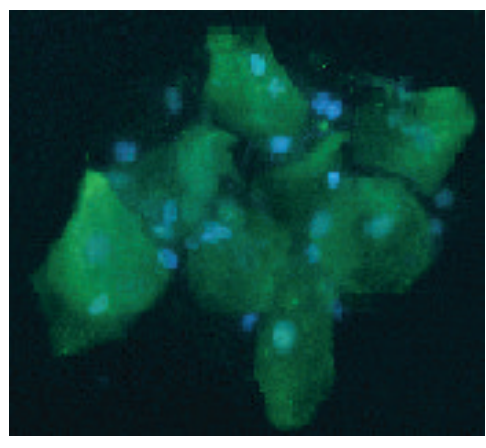

3

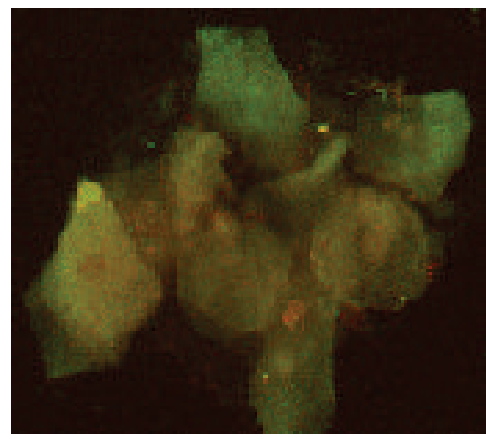

(d)

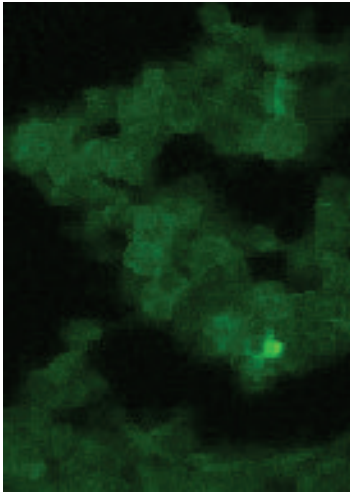

1

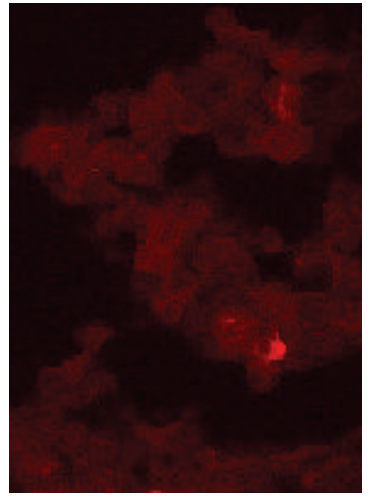

2

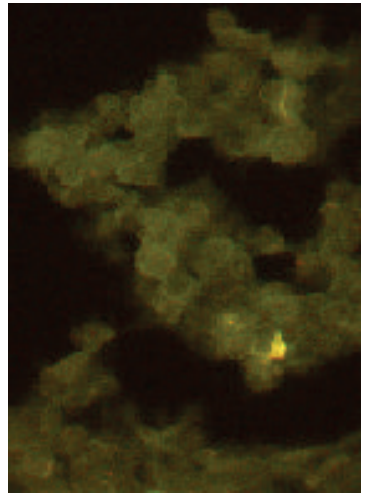

(f)

FIGURE 1: Subtelomeric signal copy number and signal intensity of Chromosome $\mathrm{X}$ in buccal cells and auxiliary lymph node. (a, b, c, d): signal mode in p- and q-arm of chromosome X in BUC cells of a patient affected with BC is presented. (a) BUC cells with phase contrast with $\times 400$ magnification; (b1) same cell population with dapi filter (blue); (b2) same cells are conjugated with FITC (p-arm in green) showing low SCN and SI; (b3) same cells conjugated with dapi and FITC reflecting a reliable image of BUC nucleus; (c1) same BUC cells conjugated with pe-cy5 (red); (c2) same cells conjugated with dapi and pe-cy5 (red), (d) conjugated BUC cells with FITC and pe-cy5 reflecting the nonharmonic behavior of $\mathrm{p}$ and q arms. (e, f, g): (e) ALN cells with dapi filter (blue); (f1) same ALN cells conjugated with FITC (p-arm in green) showing low SCN and SI; (f2) same ALN cells conjugated with pe-cy5 (red); (g) merged image of cells conjugated with FITC and pe-cy5 highlighting a diverse behavior of $\mathrm{p}$ and $\mathrm{q}$ arms. 


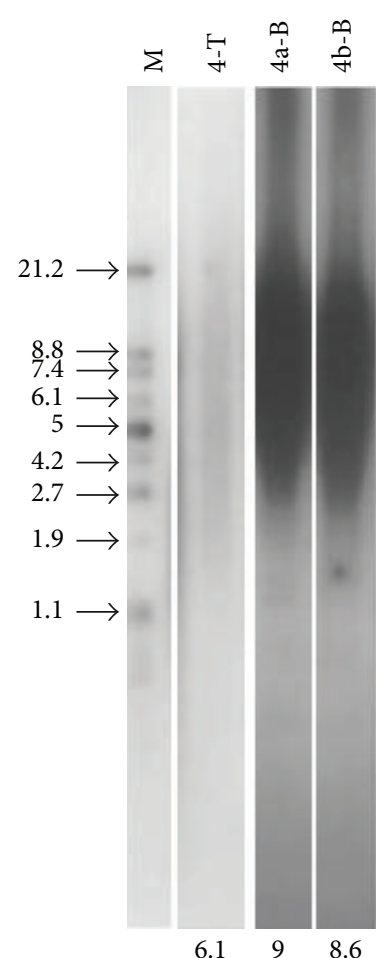

$(\mathrm{kb})$

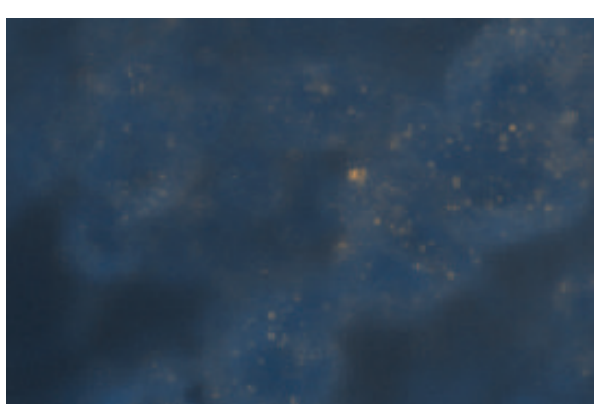

(a)

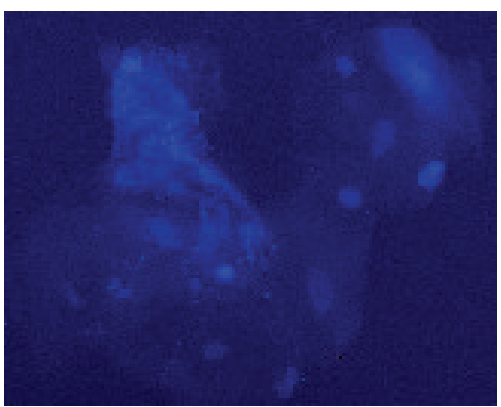

1

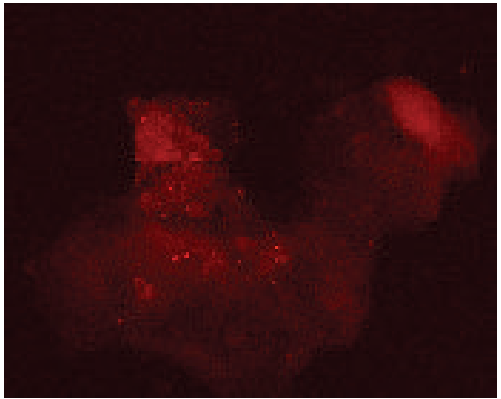

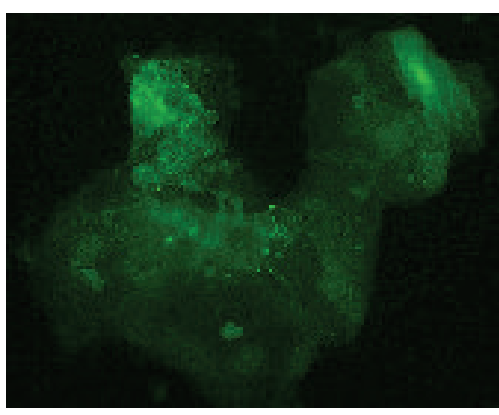

2

(b)

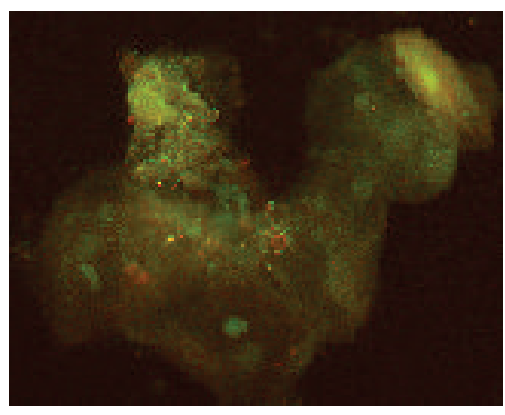

(c)

FIGURE 2: Comparative insights on telomere length in breast cancer and subtelomeric profile in auxiliary lymph node cells. Left column: TL of right breast tumor assayed by Southern blot presenting low TL at both genomics and tumor levels; (a, b, c) subtelomere statue in the left ALN and breast tumor; (a) Q-FISH image of right tumor cells conjugated with cy3(orange) and mergrd with dapi confirming low signal copy number and signal intensity of global chromosomes; (b1) BUC cells with dapi filter (in blue); (b2) same cells conjugated with FITC (in green) reflecting the signals of p-arm of chromosome 18; (c1) the same BUC cells conjugated with Pe-Cy5 (in texas red) presenting the q-arm of chromosome 18; (c2) the conjugate image of same cells presenting the signal mode of p- and q-arm. TL: telomere length; L: ladder, G: genomics; T: tumor; BUC: buccal.

categories; chromosomes 6 and 7 are predisposed to lose both normal SCN; chromosomes 8 and 10 are predisposed to lose only one SCN; chromosomes 9,11 , and 12 , besides of being involved in normal clone, are also predisposed to lose only one normal SCN or to lose either one or two SCN, to lose one SCN, respectively (Figure 9). Interestingly, by considering the decreased SCN category in ALN against BUC cells, they play different notes and scenario. Chromosomes 3, 4, 6, 7, 8, 9, 10, 18,20 , and $\mathrm{X}$ have funded the normal clone. Chromosome 1 is only involved by losing one SCN; chromosome 2 by losing either one or both SCN; chromosome 3 is involved in all categories including normal two SCN, losing either one or both SCN and also gaining an additional SCN.
Chromosomes 1, 2, 3, 6, 8, 9, 10, 11, 12, 16, 17, 20, and $\mathrm{X}$ are classified as the initial normal clone by reflecting the median intensity, comparable with control cells. Week intensity reflects downregulation (qualitative lost), and strong intensity reflects upregulation (qualitative gain). All chromosomes share the course of evolution except chromosomes 9, 10 , and $\mathrm{X}$, due to the lack of predisposition to be upregulated and downregulated, respectively (Figure 10). In upregulated mode, chromosome 18 has been participated neither for having week SI, nor strong SI which is in contrary to the downregulated status. Present data indicates that chromosomes 9 and 10 are not predisposed to be upregulated, and chromosome $\mathrm{X}$ is not capable to be downregulated. However, 


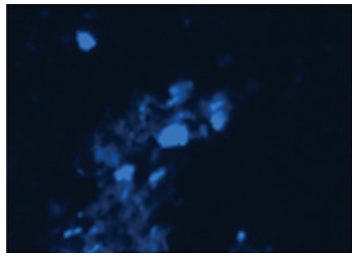

1

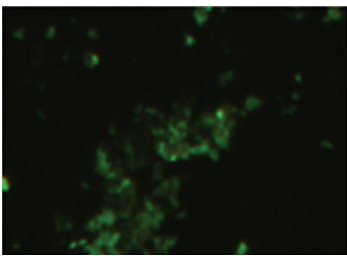

1

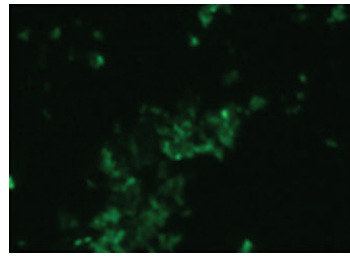

2

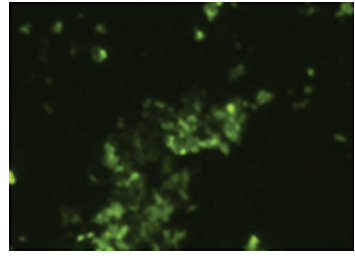

2

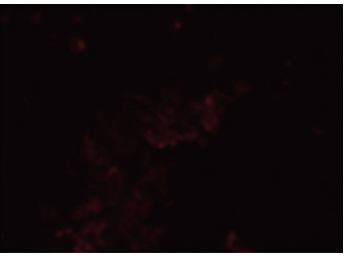

3

(a)

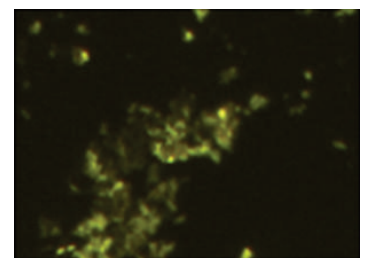

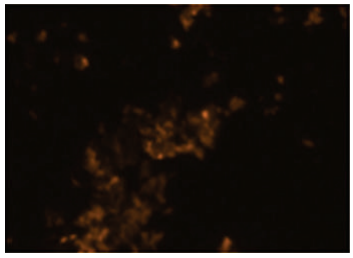

4

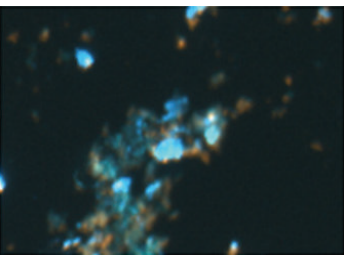

(c)

Figure 3: Protein expressions of Ki67, cyclin D1, and Cyclin E in auxiliary lymph node cells. (a1) ALN cells with dapi filter (blue), as counter stain; (a2) ALN cells conjugated with FITC (green), representative of Ki67 characterized with a minor cells with high expression by IF; (a3) same ALN cells as conjugated with Pe-Cy5 (red), representative of cyclin D1with low expression; (a4) same cells were conjugated with R-Pe (orange), representative of Cyclin E reflecting high expression in certain cell population, and harmonic expression mode in similar bunch of cells as observed in expression of Ki67; (b1) merged image of Ki67 and cyclin D1; (b2) merged image of Ki67 and cyclin E; (b3) merged image shows coexpression of Ki67, cyclin D1, and cyclin E; (c) reflects merge of dapi, Ki67, cyclin D1, and cyclin E. The merged images clarify the diverse and heterogenic expression of two key cell cycle targets; that is, cyclin D1 and cyclin E enable initiating proliferative manner; this is accompanied by the active role of Ki67 as a growth and proliferative index in the present benign neoplastic tissue, that is, ALN.

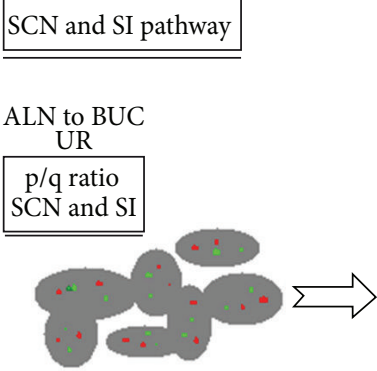

NC

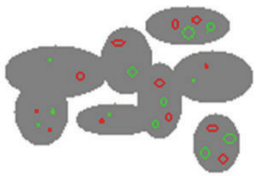

SCNL W-SI and proliferation

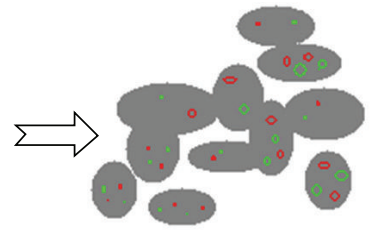

SCNL

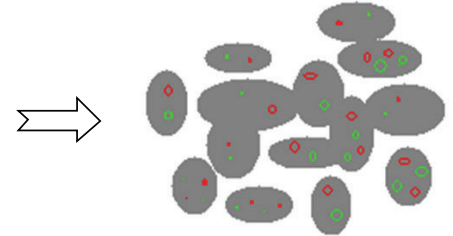

Proliferation

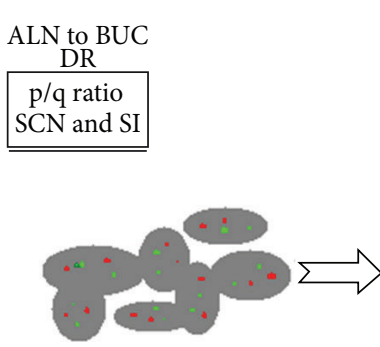

NC

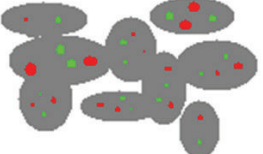

SCNL

S-SI and proliferation

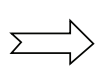

S-SI and proliferation
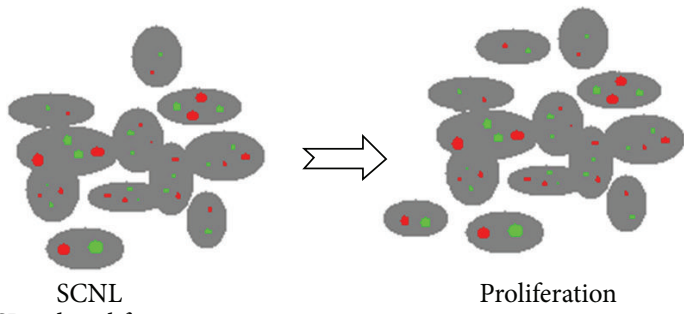

Proliferation

FIGURE 4: Subtelomeric evolutionary model for the mean ratio of p/q of chromosome 9. ALN: auxiliary lymph node; BUC: buccal; NC: normal clone; SCN: signal copy number; SCNL: losing one SCN; SI: signal intensity W: weak; M: medium; S: strong; DR: downregulated; UR: upregulated evolutionary pattern is diverse in categories "ALN to BUC-UR" and "ALN to BUC-DR" which are reflective of "p/q ratio being significantly higher in ALN than in buccal cells" and "p/q ratio being significantly lower in ALN than in buccal cells," respectively. 


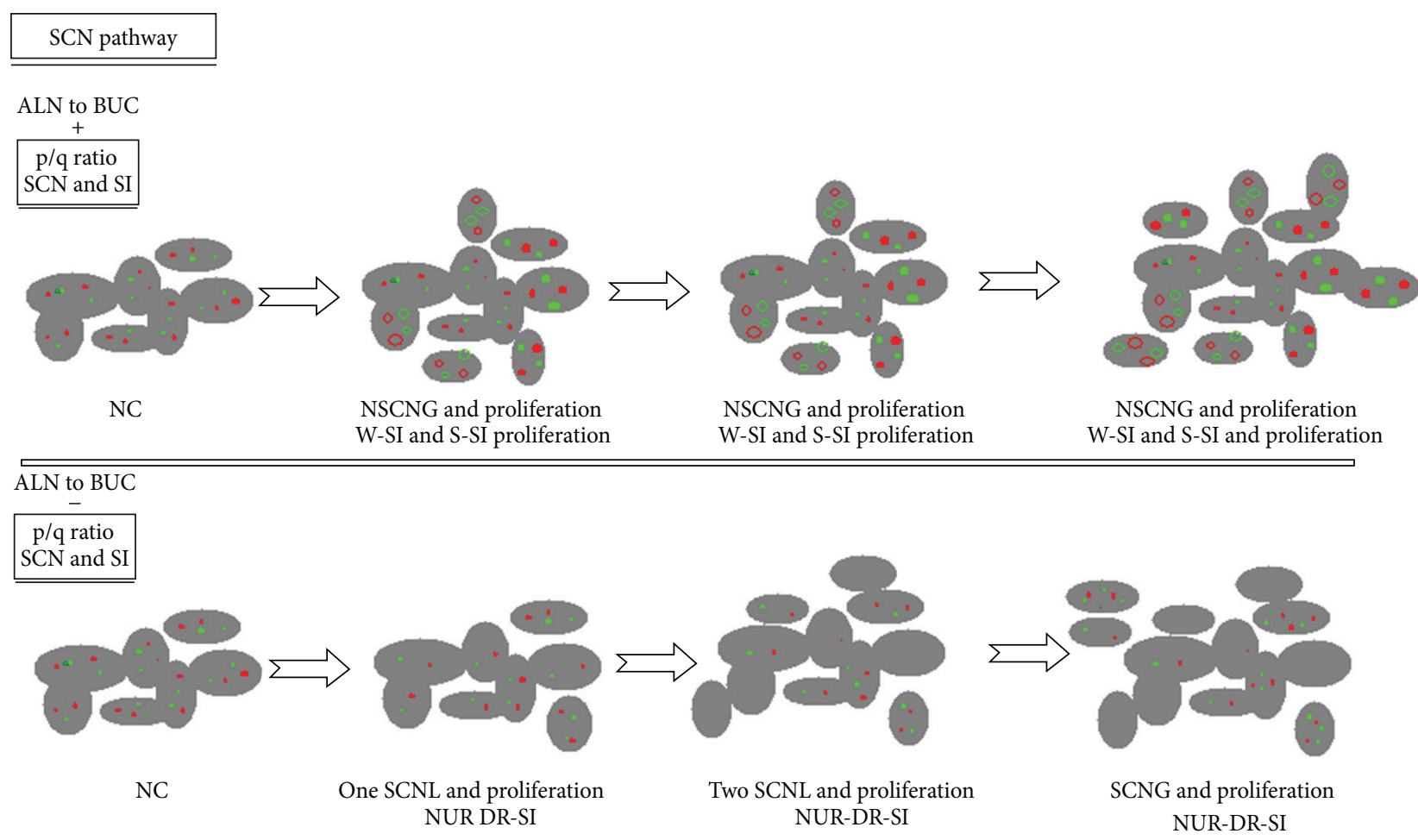

NUR-DR-SI: no up-regulated-down-regulated SI

FIGURE 5: Subtelomeric evolutionary model for the mean ratio of p/q of chromosome 5. ALN: auxiliary lymph node; BUC: buccal, NC: normal clone; SCN: signal copy number; SCNL: losing one SCN; SI: signal intensity, W: weak; M: medium; S: strong; DR: downregulated; UR: upregulated. Evolutionary pattern is diverse in categories "ALN to BUC-UR" and "ALN to BUC-DR" which are reflective of "p/q ratio being significantly higher in ALN than in buccal cells" and "p/q ratio being significantly lower in ALN than in buccal cells", respectively. NUR-DR-SI: no up-regulated-down-regulated SI.

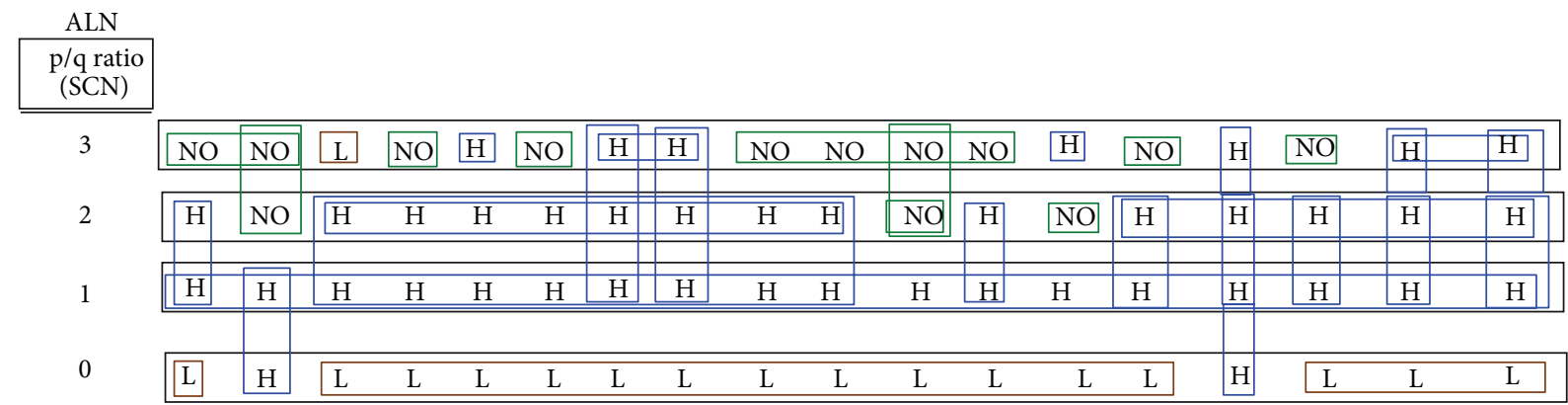

\begin{tabular}{|c|c|c|c|c|c|c|c|c|c|c|c|c|c|c|c|c|}
\hline Chromosomes 1 & 2 & 3 & 4 & 6 & 7 & 8 & 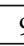 & 1( & 11 & 12 & 16 & 17 & 18 & 19 & 20 & $X$ \\
\hline
\end{tabular}

\begin{tabular}{|c|c|c|c|c|c|c|c|c|c|c|c|c|c|c|c|c|c|c|}
\hline 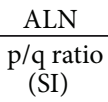 & & & & & & & & & & & & & & & & & & \\
\hline W & (I) & $\mathrm{H}$ & $\overline{\mathrm{H}}$ & $\mathrm{H}$ & $\mathrm{H}$ & $\mathrm{H}$ & $\overline{\mathrm{H}}$ & $\mathrm{H}$ & $\overline{\mathrm{H}}$ & $\mathrm{H}$ & $\mathrm{H}$ & $\mathrm{H}$ & & $\mathrm{H}$ & $\mathrm{L}$ & $\mathrm{H}$ & $\mathrm{H}$ & $\mathrm{H}$ \\
\hline $\mathrm{M}$ & \begin{tabular}{|l|}
$\mathrm{L}$ \\
\end{tabular} & $\mathrm{H}$ & $\mathrm{H}$ & $\mathrm{H}$ & \begin{tabular}{|l|}
$\mathrm{L}$ \\
\end{tabular} & $\mathrm{H}$ & $\mathrm{H}$ & $\mathrm{H}$ & $\mathrm{H}$ & $\mathrm{H}$ & $\mathrm{H}$ & $\mathrm{H}$ & & $\mathrm{H}$ & $\mathrm{L}$ & $\mathrm{H}$ & $\mathrm{H}$ & $\mathrm{H}$ \\
\hline & & & & & & & & & & & , & 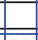 & & 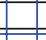 & & . & . & \\
\hline$S$ & $\mathrm{H}$ & $\mathrm{H}$ & $\mathrm{H}$ & $\mathrm{H}$ & $\mathrm{H}$ & $\mathrm{H}$ & $\mathrm{H}$ & $\mathrm{H}$ & $\mathrm{H}$ & $\mathrm{NO}$ & $\mathrm{H}$ & $\mathrm{H}$ & $\mathrm{H}$ & $\mathrm{H}$ & $\mathrm{H}$ & $\mathrm{H}$ & $\mathrm{H}$ & $\mathrm{H}$ \\
\hline
\end{tabular}

FIGURE 6: Distribution of p/q ratio of subtelomeric signal copy number and signal intensity in auxiliary lymph node cells per individual chromosomes. L: low; H: high; p/q ratio<1: L; p/q-ratio>1: H; NO: not observed; left column: different categories of SCN and SI; 0: lacking SCN; 1: losing one SCN; 2: normal clone by having 2 SCN, 3: gaining one SCN; W: weak intensity; M: medium intensity, S: strong intensity. 


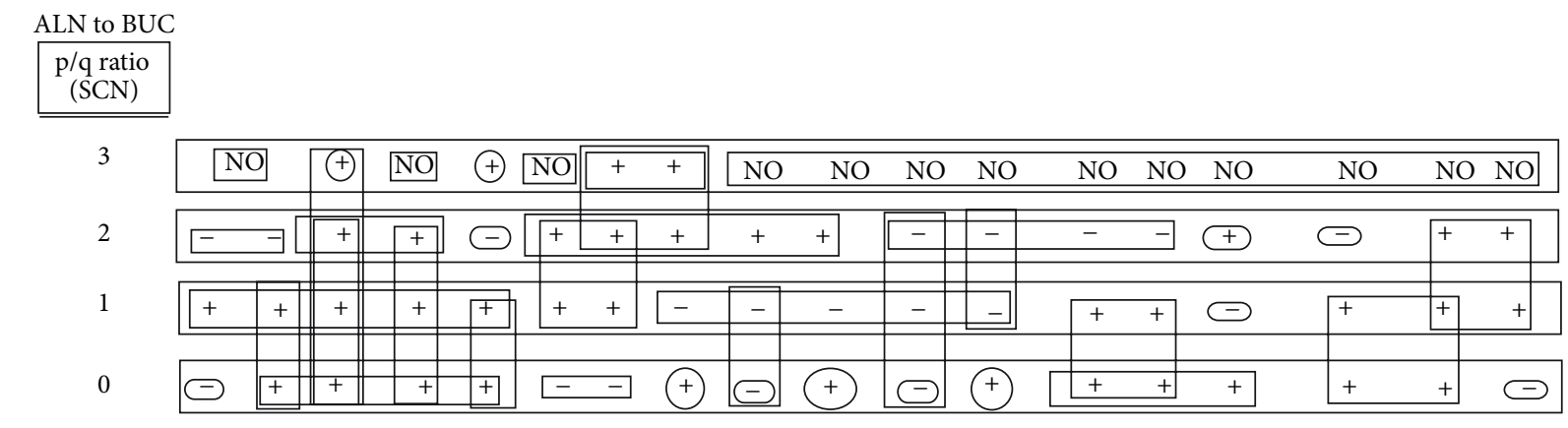

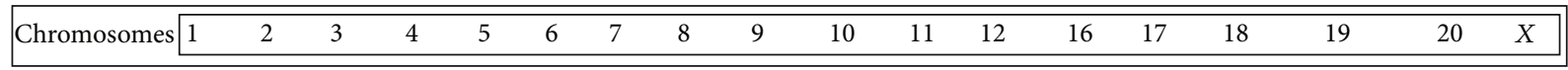

\begin{tabular}{|c|c|c|c|c|c|c|c|c|c|c|c|c|c|c|c|c|c|c|}
\hline $\begin{array}{c}\mathrm{p} / \mathrm{q} \text { ratio } \\
\text { (SI) }\end{array}$ & & & & & & & & & & & & & & & & & & \\
\hline$S$ & UR & $\overline{\text { UR }}$ & $\overline{\overline{U R R}}$ & UR & UR & UR & UR & UR & DR & $\mathrm{DR}$ & UR & UR & UR & UR & $\mathrm{DR}$ & UR & UR & UR \\
\hline M & UR & UR & UR & \begin{tabular}{|l}
$\mathrm{DR}$ \\
\end{tabular} & $\overline{\mathrm{DR}}$ & UR & DR & UR & UR & UR & UR & UR & UR & UR & DR & DR & UR & UR \\
\hline W & UR & UR & UR & UR & UR & UR & UR & UR & UR & UR & UR & UR & UR & UR & $\mathrm{DR}$ & UR & UR & DR \\
\hline
\end{tabular}

FIGURE 7: Distribution of p/q ratio of subtelomeric signal copy number and signal intensity in buccal and auxiliary lymph node cells per individual chromosomes, +: p/q ratio of SCN is increased in ALN; -: p/q ratio of SCN is decreased in ALN; UR: upregulated; DR: downregulated; NO: not observre; left column: different categories of SCN and SI; 0: lacking SCN; 1: Losing one SCN; 2: normal clone by having 2 SCN; 3: gaining one SCN; W: weak intensity; M: medium intensity; S: strong intensity. The common subtelomeric categories are vertically and horizontally framed.

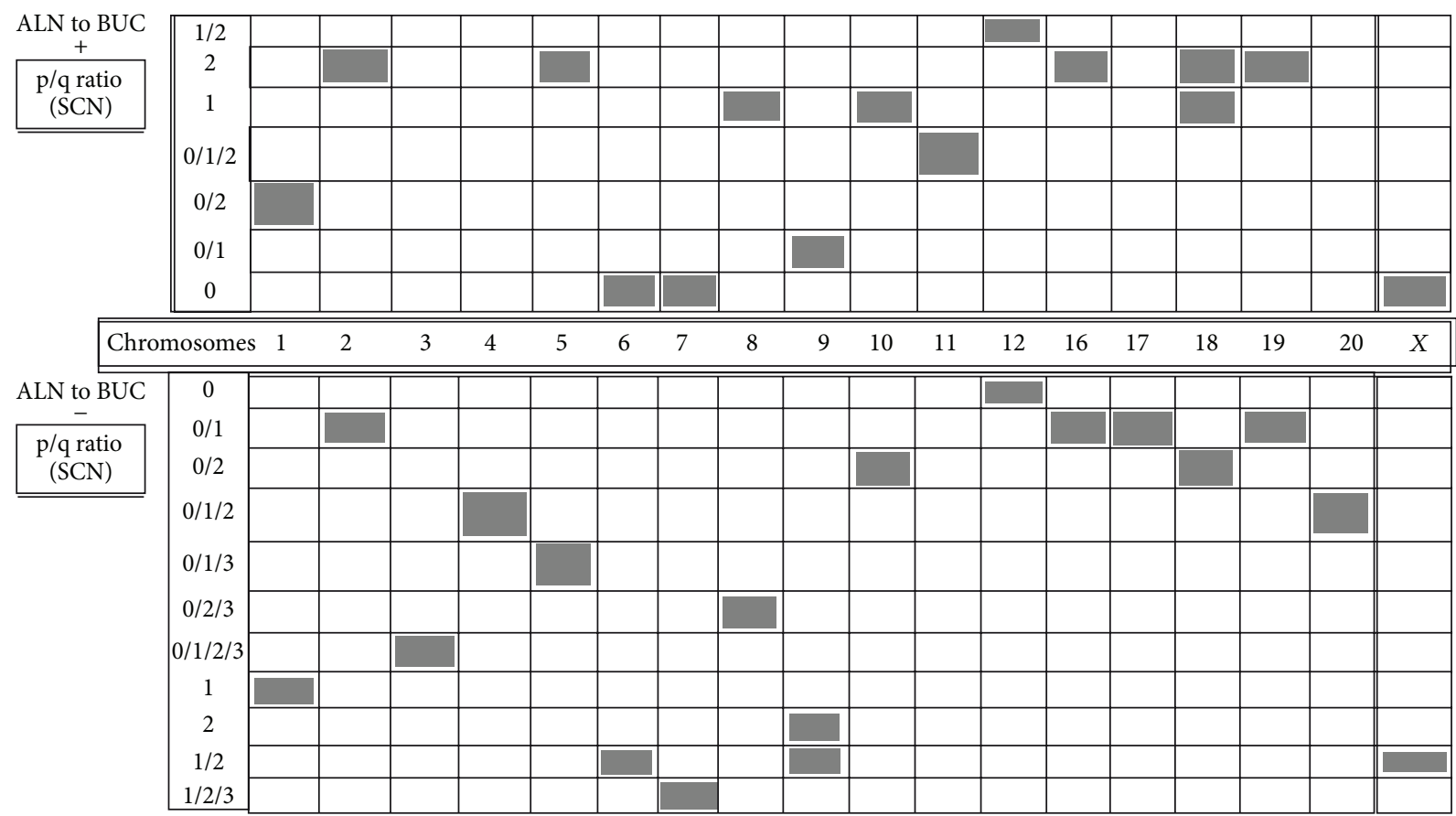

FIGURE 8: Distribution of p/q ratio of subtelomeric signals copy number, based on evolutionary alteration within the buccal- and auxiliary lymph node-cells and different clones, per individual chromosomes; BUC: buccal; NO: not observed, +: p/q ratio of SCN is increased in ALN; $-: \mathrm{p} / \mathrm{q}$ ratio of SCN is decreased in ALN left column present different cell populations: 0: lacking SCN; 1: losing one SCN; 2: normal clone by having 2 SCN; 3: gaining one SCN. 


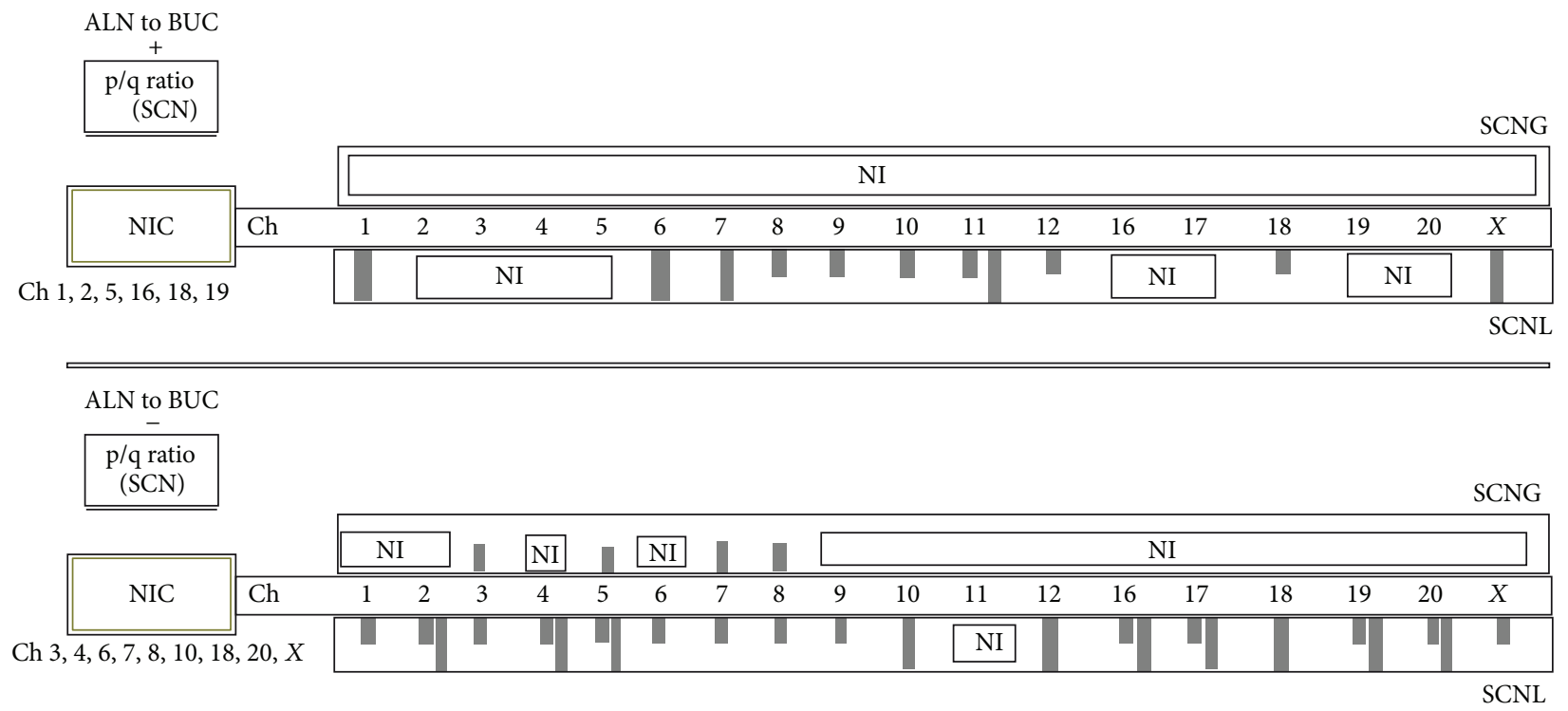

FIGURE 9: Evolutionary periodic chart of subtelomeric signal copy number through buccal- and auxiliary lymph node cells-Chromosomes $2,5,16,17,19$ are classified as being involved in normal clone. +: p/q ratio of SCN is increased in ALN. -: p/q ratio of SCN is decreased in ALN; NIC: normal initial clone having 2 SCN; SCNG: signal copy number gain; SCNL: signal copy number loss; NI: not involved, Greyish half column: losing one signal, Greyish full column: losing two signals.

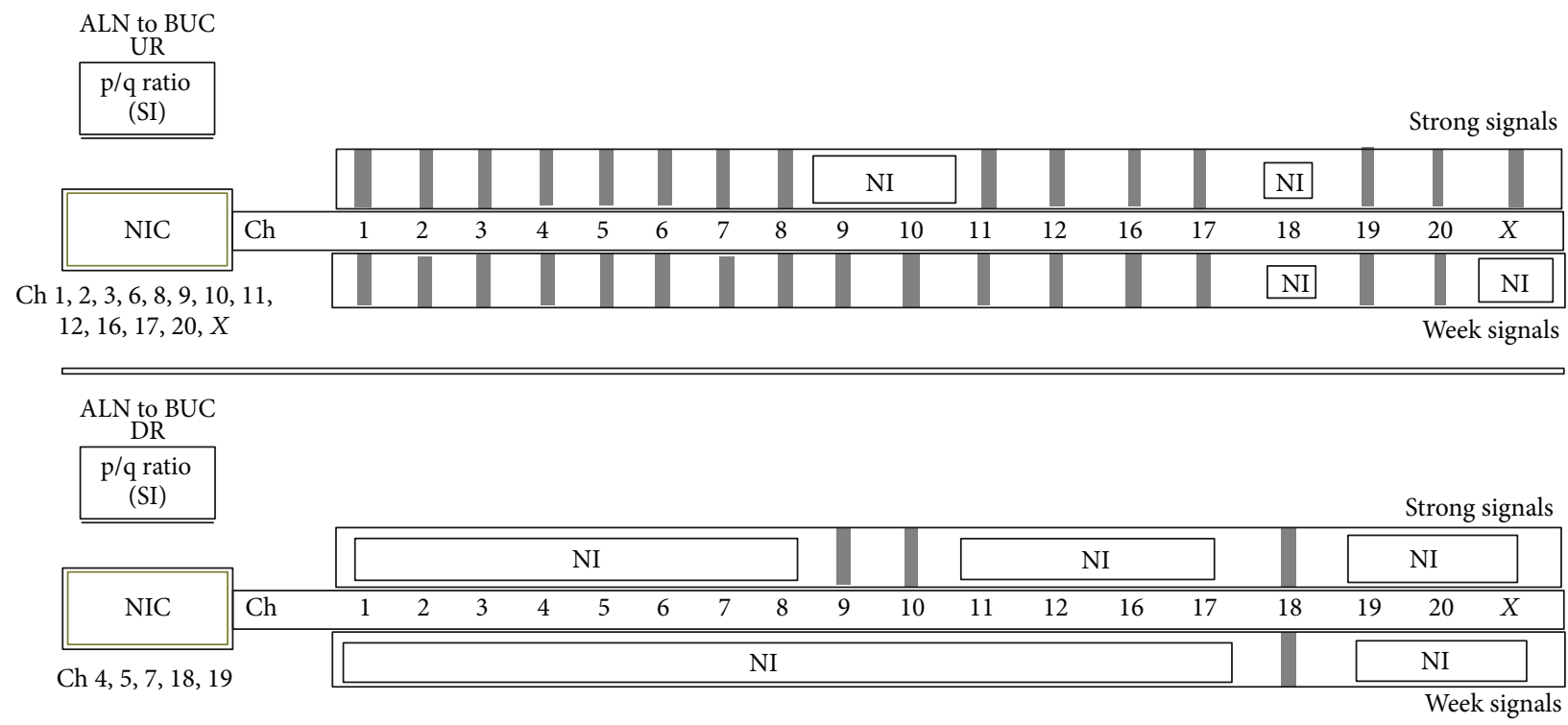

FIGURE 10: Evolutionary Periodic chart of subtelomeric signal intensity, based on evolutionary alteration within the buccal- and auxiliary lymph node-cells and different clones, per individual chromosomes. NO: not observed; BUC: buccal, DR: downregulated; UR: upregulated; NI: not involved; NIC: Normal initial clone having 2 SCN; M: medium as normal signal intensity; W: weak intensity reflects downregulation (qualitative loss); S: strong intensity reflects upregulation (qualitative gain); Greyish full column is indicative of the involved chromosomes.

chromosome 18 is not predisposed to SI alteration and does not participate in the course of evolution (Figure 10).

By losing two SCN, it is expected to detect no SI; this is, relatively, true when the SI of chromosome is identified within the downregulated category (Figures 9 and 10). But at the same figures it is noticeable that there is a strong SI in upregulated row; in this case, it is valuable to check the karyotype of tissue and trace even a minor clone with partial or whole trisomy of X-chromosome.
Figures 4 and 5 are considered as two evolutionary models for subtelomeric SCN and SI for individual chromosomes which announce the subtelomeric behavior. However, to provide more specific and personalized models for each chromosome, reflection of the most characteristic course of evolution would be possible. In this regard, the evolutionary model based on the p/q ratio for subtelomeric SCN and SI of chromosomes 9 and 5 is, respectively, presented (Figures 4 and 5). As it is shown, when $\mathrm{p} / \mathrm{q}$ ratio is increased in 
ALN-cells than in BUC-cells (category 1), there is no sign of SCN gain and strong SI in chromosome 9; instead, developmental process include loss of one SCN and occurrence of downregulated SI as week SI (Figure 4). In addition, when subtelomeric behavior seems to be decreased in ALN than in BUC cells (category 2), losing one SCN is similar to the previous category, but interestingly, strong SI is indicative of upregulated status of SI (Figure 4). It could be concluded that in category 1 , the course of evolution affects normal clone by further process including loss of one SCN, accompanied by downregulating of signal qualitative mode "as week SI" and proliferation. But in category 2, the loss of SCN is accompanied by upregulated event, that is, strong SI. This event reflects the heterogenetic behavior of ALN cells which also depends on the diverse ratio of $\mathrm{p} / \mathrm{q}$ individual chromosomes' arms. This fact announces an evolutionary message at BUC/ALN level.

By looking at chromosome 5 , the scenario is completely different, in category 1; there is no sign of SCN loss and gain with both week and strong SI, but in category 2; loss and gain of one signal, loss of two SCN, and no further development of SI including weak and strong could be observed (Figure 5). As conclusion, the nature of evolution in category 1 is revealed to be no SCN gain, accompanied by both up- and downregulation of quantitative value as weak- and strongSI and proliferation. But in category 2, evolutionary process from normal clone is led to proliferative neoplasm by losing one-, two-SCN, gaining one SCN and no sign of up- and down-regulation of signal intensity (Figure 5). As an example, there is a remarkable diversity between chromosomes 5 and 9. This is true for other chromosomes as well (Figures 7 and 8).

Different chromosomes either play their own instrumental notes or play harmonic. If loss of SCN is accompanied by strong SI (upregulated mode), it may be due to diverse behavior of one or more chromosome(s) in different cells which may lead to the heterogenic function at qualitative and quantitative levels. Furthermore, by considering $\mathrm{p} / \mathrm{q}$ ratio for an individual BC or an apparently healthy individual, 18 evolutionary model could be provided, and then grouping of each common models would be possible. So, what matters in cancer is diversity, not only in individual cells but also in specific chromosome(s). Collectively, these findings could be considered as the evolutionary cascade and with a complementary karyotyping test a new direction would be opened to unmask more complex interaction with chromosomal fragile sites and discovering the new gene(s). In addition, according to the logistic regression, ALN was considered as a variable referring factor.

Moreover, the genomics telomere length (TL) of this patient has been previously assayed and revealed to be $9.00 \mathrm{~kb}$ and $8.60 \mathrm{~kb}$ when she was 57 and 66 years, respectively. The tumor TL was $6.1 \mathrm{~kb}$ and the TL ratio for genomic/ALN is reported to be 1.47 at age of 57 and 1.40 at age of 66 . Telomerase activity was positive [16] (Figure 2). Collectively, the package of complementary information on TL and ST would also be able to clarify aging and cancer cell as a united insight.

\section{Protein Expression}

Status of protein expression for Ki67, cyclin D1, and cyclin E on ALN was assayed by IF (Figure 3). Although the nature of ANL is found to be benign, a minor clone of cells reflect relatively high expression for Ki67 and cyclin E which may reflect a hyperplasic mode for few ALN cells, and in spite of the low expression of cyclin D1, cyclin E has played an effective role in the cell cycle process. As the progressive cell cycle machinery indicates, the diverse and heterogenic expression of cyclin D1 and cyclin E is remarkable in the diagnosed ALN as an apparently benign tissue. Furthermore, the approving role of Ki67 provide rising of a new developmental insight within the ALN platform. There is a great concern on the benign morphological appearance of ALN cells.

\section{Conclusions}

The system biology of cancer cells is rather complex and confusing due to the multidisciplinary machinery. In this regard, laddering and mining insights may be useful to unmask some facts within the territory which have the influential impacts on aging and carcinogenesis. The nature of mechanisms in subtelomeric domain is not clear and the facts at cellular level may lead to achieving the manner of some key pathways.

Novel Periodic charts for subtelomeric quantitative and qualitative values could provide a personalized buccal- and auxiliary lymph node-cell based profile at the somatic level.

By considering the $\mathrm{p} / \mathrm{q}$ ratio for subtelomeric profile in BUC-cells and ALN, different Periodic charts would facilitate the laddering status of signal copy number and signal intensity.

Individualized cell based subtelomeric profile will lead to design a reliable signature of subtelomeric $\mathrm{p} / \mathrm{q}$ ratio for SCN and SI in BUC and ALN cells. Such pathways could lead to unmask more specific molecular diversity in specific chromosomes' arm through mining different clone of cells and tracing undiscovered gene(s).

Regarding diversity and heterogeneity aspects, decreased and elevated p/q ratio of SCN and mode of regulation of signal intensity could be translated from BUC destination to the ALN platform.

Periodic charts are considered as the informative and refreshable platforms through which the evolutionary model of subtelomeric SCN through BUC- and ALN-cells could be designed in different aspects of cell biology.

By considering the $\mathrm{BUC}$ as a reliable and index biologic target, increased and decreased SCN in ALN cells could be identified as the result of an evolutionary course.

Evolutionary model of subtelomeric SI through these two cell populations provide a platform in which these alterations would be traceable at individual chromosomes' arms. Finally, besides the key role of subtelomeric behavior, the complementary and approving role of Ki67 initiate a new developmental insight within the ALN platform. As the final words, telomere, telomerase, and subtelomere, as the cellular marker, play complementary role in aging cell and neoplasm. 


\section{Materials and Methods}

\subsection{Materials}

6.1.1. Patient's Information. The left auxiliary lymph node (ALN) of a 66-year-old female who was previously affected with infiltrative ductal carcinoma of right breast at age of 57years was negative for malignancy. The patient had a positive family history of cancer including the breast.

Total cell of 2309 and 38256 was analyzed at BUC and ALN level, respectively. The ALN was, subtelomerically, assayed. To deem the ethics regulations, the consent letter was signed by her.

6.1.2. Subtelomere Multiprobe. The Cytocell subtelomere specific probe "The Chromoprobe Multiprobe" is used (http:// www.cytocell.com/). This system facilitates the application of multiple FISH probes by considering the whole set of chromosomes at the same time and condition. The width capacity of this subtelomeric probe is to scan the "subtelomere enumeration and integrity" (http://www.cytocell.com/). The $\mathrm{p}$ and $\mathrm{q}$ probes are conjugated with FITC and pe-cy5, respectively, for the same chromosome, and the signals are visible by fluorescence microscope (Leica Co., Germany). This Multiprobe is located in the most distal region of chromosome specific DNA, with the average size of $100 \mathrm{~kb}$, and the clones on the Chromoprobe Multiprobe-T System are mapped to within $600 \mathrm{~kb}$ of the true telomere. Each of 24 Multiprobe devices contains the specific probe for both the $\mathrm{p}$ arm and q-arm of each chromosome.

\subsection{Methods}

6.2.1. Cell Preparation. Cell populations included buccal (BUC)-cells as an internal control tissue, and auxiliary lymph node (ALN)-cells as the somatic-neoplastic platform. Buccalcells were isolated through washing process by Phosphate buffer solution (PBS) twice, followed by fixation in $3 / 1$ mixture of methanol/glacial acetic acid. Five-micrometerthick paraffinized tissue of auxiliary lymp node was placed on the positively charged slide and deparaffinized with xylene twice, then rehydrated with $100 \%$ ethanol, air-dried, and treated with pepsin. The cells were washed in PBS twice and fixed in 3/1 mixture of methanol/glacial acetic acid. The pand $\mathrm{q}$-arm probes are labeled with a fluorophore specific for the spectrum of FITC and Pe-Cy5 (Texas red).

\subsubsection{Fluorescence In Situ Hybridization (FISH)}

(i) Template Slide Spotting

(a) Density of cells on template slide is required to be confirmed by $\times 10$ objective.

(b) Pipetting $2 \mu \mathrm{L}$ of cell suspension onto all 24 areas.

(ii) Pretreatment of Slide

(a) Template slide is washed in $2 \times$ SSC. (b) Template slide is dehydrated through an ethanol series at $70 \%-100 \%$ ethanol and placed att $37^{\circ} \mathrm{C}$.

(c) Mix the hybridization solution of $30 \mu \mathrm{L}$ aliquot per Multiprobe device in $37^{\circ} \mathrm{C}$ incubator.

(d) Add $1 \mu \mathrm{L}$ of hybridization solution to each of the Multiprobe devices.

(iii) Denaturation. The slide/Multiprobe device was placed at $75^{\circ} \mathrm{C}$ for 2 minutes.

(iv) Hybridisation. The slide is placed in the $37^{\circ} \mathrm{C}$ water chamber overnight.

(v) Posthybridization Process

(a) The slide is treated with $0.4 \times$ SSC solution at $72^{\circ} \mathrm{C}$ for 2 minutes.

(b) The slide is placed in the $2 \times \mathrm{SSC} / 0.05 \%$ tween solution, for 30 seconds.

(vi) Mounting and Visualization

(a) Adding $20 \mu \mathrm{L}$ of DAPI-Antifade Solution to the slide and cover with $24 \mathrm{~mm} \times 60 \mathrm{~mm}$ Coverslip.

It is expected that all chromosomes except the acrocentric D \& G groups, as a normal sample, reflect 2 green ( $\mathrm{p}$-arm) and 2 red (q-arm) signals. Complete deletion of subtelomere regions will lead to the absence of signal, and the signal intensity is classified as weak, medium, and strong.

6.3. Immunofluorescence (IF). IF was performed to detect the mode of protein expression at the cellular level, according to the previously provided technique $[16,17]$.

The cells were processed by monoclonal mouse antihuman Ki67 (FITC conjugated,isotype IgG1, Dako Cytomation), anti-cyclin D1 (IgG2a isotype, Biosource), and mouse anti-cyclin E (isotype IgG2b, Zymed Laboratories, Invitrogen immunodetection).

Range of 1000-5000 cells was washed with $1 \mathrm{x}$ PBS, hybridized with antibody, and incubated at 4 degree $\mathrm{C}$ for 25 minutes, followed by a further washing with $1 \mathrm{x}$ PBS. The corresponding anti-mouse is added and incubated at 4 degree $\mathrm{C}$ for 25 minutes. Finally, the nature of protein expression was detected by the LEICA, DM RXA2-fluorescence microscope.

6.4. Telomere Length Assays. Southern blot analysis was performed according to the provided standard technique by Roche diagnostics. As a complementary test, the Q-FISH was assayed by Telomere PNA FISH Kit/Cy3, code K5326, Dako, Denemark (http://www.dako.com/ar42/p107840/prod_products.htm).

6.5. Data Analyses. Database was organized with SPSS version 17. The statistical tests are as follows.

The results were provided as mean \pm standard deviation. EM-algorithms (Expectation Maximization) were applied to achieve a reliable standard deviation (SD) for stabilizing the mean of for individual chromosomes. 
(i) One-way ANOVA test is considered to present diversity between frequencies of individual chromosomes and the target factors.

(ii) Pearson correlation coefficient test was used to achieve the correlations between the relevant factors. This was endorsed on the basis of central limit theorem.

(iii) Binary logistic regression is analyzed for the crucial regression models. levels.

Significance was considered at $P<0.001$ and $P<0.05$

\section{Abbreviations}

ALN: Auxiliary lymph node

BC: $\quad$ Breast cancer

BUC: Buccal

FISH: Fluorescence in situ hybridization

IF: Immuno-fluorescence

SCN: Signal copy number

SI: $\quad$ Signal intensity

ST: $\quad$ Subtelomere

Q-FISH: Quantitative fluorescence in situ hybridization.

\section{Conflict of Interests}

All authors disclose that there are no commercial affiliations or consultancies, stock or equity interests, or patent-licensing arrangements that could be considered a conflict of interests regarding the submitted paper.

\section{Acknowledgment}

The authors appreciate the great assistance of Ms. Baghdasarian during the sampling process.

\section{References}

[1] G. Taybos, "Oral changes associated with tobacco use," The American Journal of the Medical Sciences, vol. 326, no. 4, pp. 179$182,2003$.

[2] T. de Lange, L. Shiue, R. M. Myers et al., "Structure and variability of human chromosome ends," Molecular and Cellular Biology, vol. 10, no. 2, pp. 518-527, 1990.

[3] S. Steinert, J. W. Shay, and W. E. Wright, "Modification of subtelomeric DNA," Molecular and Cellular Biology, vol. 24, no. 10, pp. 4571-4580, 2004.

[4] International Human Genome Sequencing Consortium, "Finishing the euchromatic sequence of the human genome," Nature, vol. 431, pp. 931-945, 2004.

[5] H. Riethman, A. Ambrosini, C. Castaneda et al., "Mapping and initial analysis of human subtelomeric sequence assemblies," Genome Research, vol. 14, pp. 18-28, 2004.

[6] H. der Sarkissian, S. Bacchetti, L. Cazes, and J. A. LondoñoVallejo, "The shortest telomeres drive karyotype evolution in transformed cells," Oncogene, vol. 23, no. 6, pp. 1221-1228, 2004.

[7] H. C. Mefford and B. J. Trask, "The complex structure and dynamic evolution of human subtelomeres," Nature Reviews Genetics, vol. 3, no. 2, pp. 91-102, 2002.
[8] E. V. Linardopoulou, E. M. Williams, Y. Fan, C. Friedman, J. M. Young, and B. J. Trask, "Human subtelomeres are hot spots of interchromosomal recombination and segmental duplication," Nature, vol. 437, no. 7055, pp. 94-100, 2005.

[9] M. K. Rudd, C. Friedman, S. S. Parghi, E. V. Linardopoulou, L. Hsu, and B. J. Trask, "Elevated rates of sister chromatid exchange at chromosome ends," PLoS Genetics, vol. 3, no. 2, article e32, 2007.

[10] S. M. Gadalla, R. Cawthon, N. Giri, B. P. Alter, and S. A. Savage, "Telomere length in blood, buccal cells, and fibroblasts from patients with inherited bone marrow failure syndromes," Aging, vol. 2, pp. 867-874, 2010.

[11] L. T. M. Dórea, J. R. C. Meireles, J. P. R. Lessa et al., "Chromosomal damage and apoptosis in exfoliated buccal cells from individuals with oral cancer," International Journal of Dentistry, vol. 2012, Article ID 457054, 6 pages, 2012.

[12] D. J. Wolff, K. Clifton, C. Karr, and J. Charles, "Pilot assessment of the subtelomeric regions of children with autism: detection of a 2q deletion," Genetics in Medicine, vol. 4, no. 1, pp. 10-14, 2002.

[13] P. Thomas, N. J. O'Callaghan, and M. Fenech, “Telomere length in white blood cells, buccal cells and brain tissue and its variation with ageing and Alzheimer's disease," Mechanisms of Ageing and Development, vol. 129, no. 4, pp. 183-190, 2008.

[14] J. Lee, X. Lu, E. S. Shin, W. F. Kern, J. J. Mulvihill, and S. Li, "A novel subtelomeric translocation $\mathrm{t}(5 ; 9)$ and a deletion of the RB1 gene in a patient with acute myeloid leukemia (AML-M0)," Cancer Genetics and Cytogenetics, vol. 181, no. 1, pp. 36-39, 2008.

[15] S. Tosi, S. W. Scherer, G. Giudici, B. Czepulkowski, A. Biondi, and L. Kearney, "Delineation of multiple deleted regions in $7 \mathrm{q}$ in myeloid disorders," Genes, Chromosomes \& Cancer, vol. 25, pp. 384-392, 1999.

[16] P. Mehdipour, M. Kheirollahi, M. Mehrazin, N. Kamalian, and M. Atri, "Evolutionary hypothesis of telomere length in primary breast cancer and brain tumour patients: a tracer for genomictumour heterogeneity and instability," Cell Biology International, vol. 35, no. 9, pp. 915-925, 2011.

[17] P. Mehdipour, Telomere Territory and Cancer, Springer, Amsterdam, The Netherlands, 2013. 


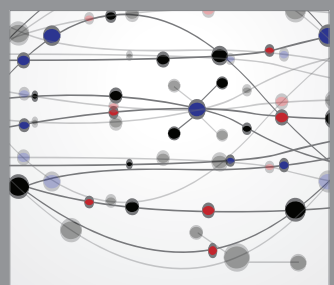

The Scientific World Journal
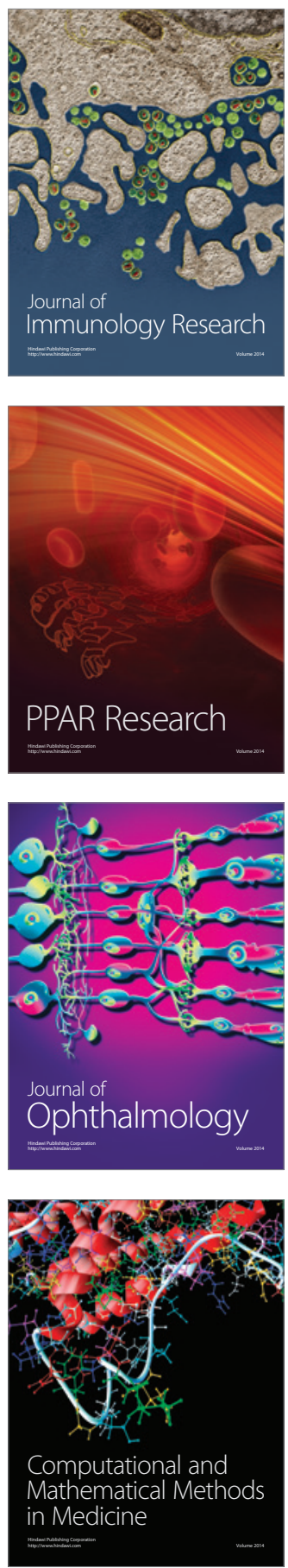

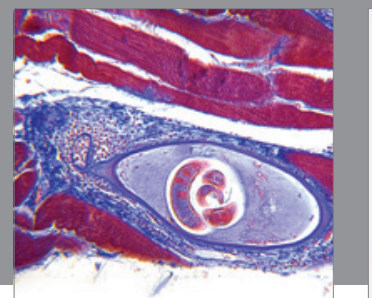

Gastroenterology

Research and Practice
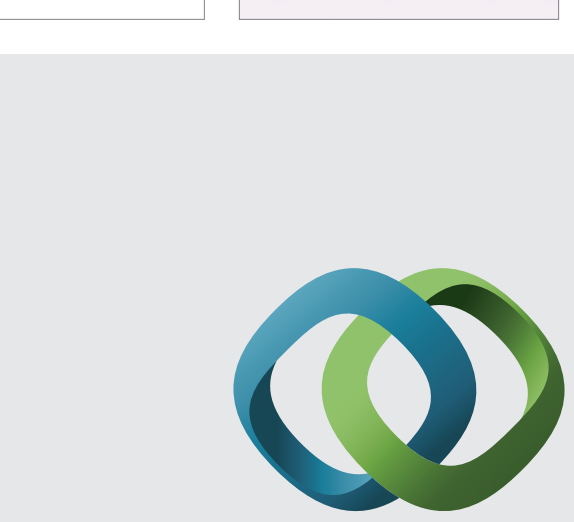

\section{Hindawi}

Submit your manuscripts at

http://www.hindawi.com
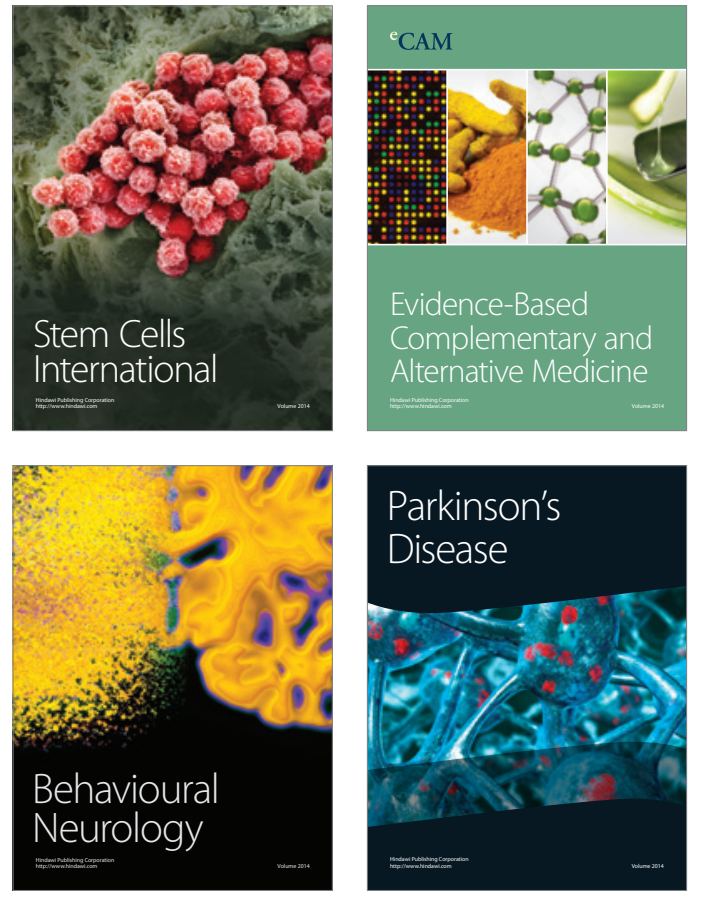
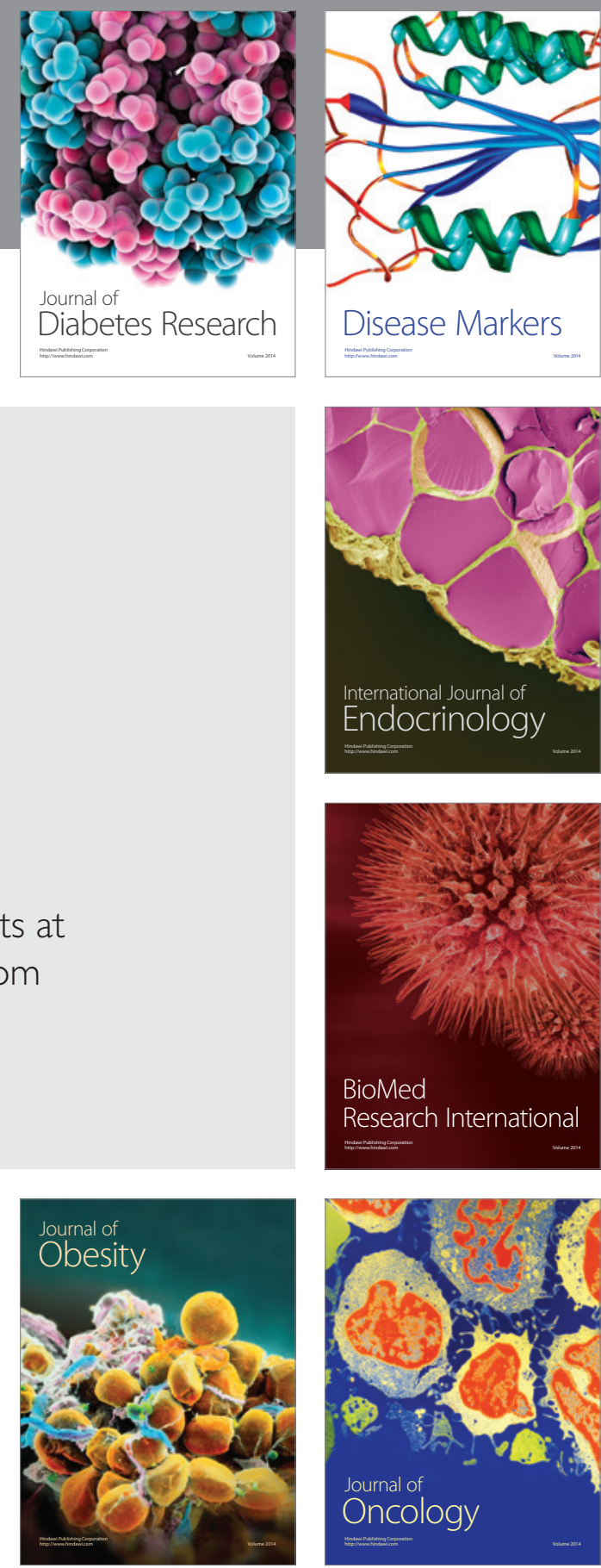

Disease Markers
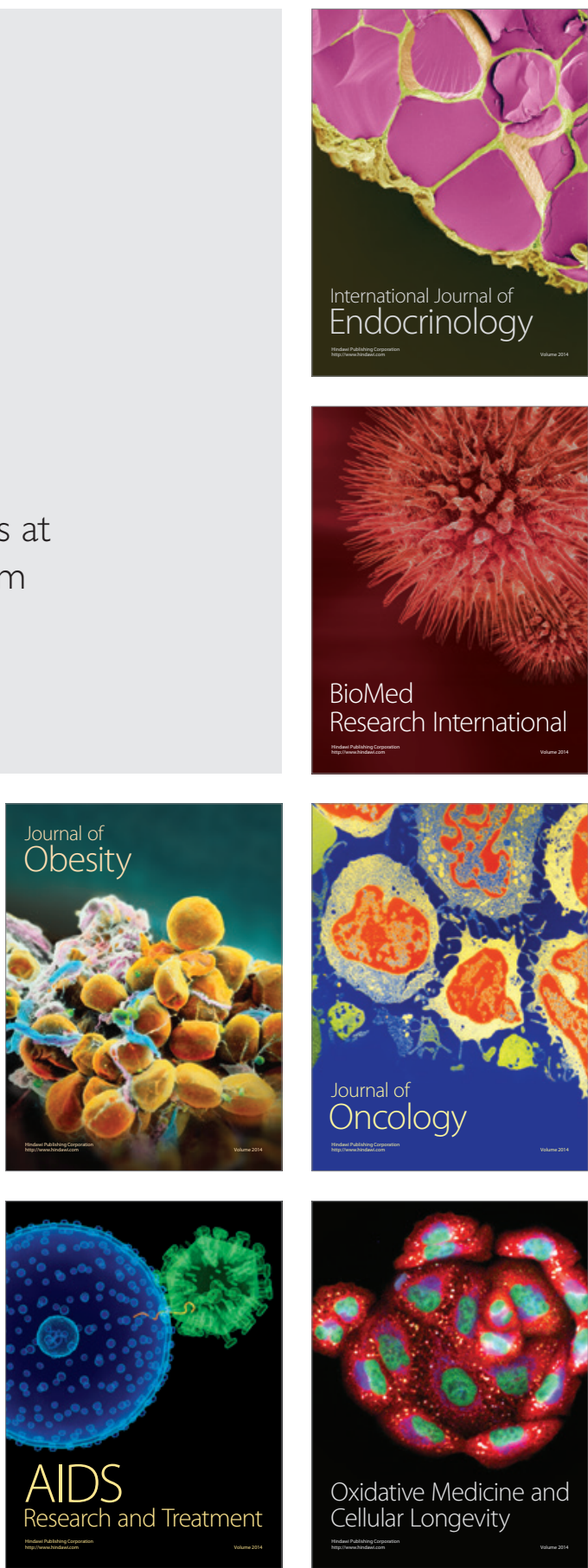